\title{
Inhalisübersicht
}

\section{SCHWERPUNKT: Steuerung digitale Geschäftsmodelle - Warum effektives Controlling erfolgskritisch ist}

Management und Controlling der digitalen Wertschöpfung

Prof. Dr. Wolfgang Becker

\section{Steuerung eines digitalen Vertriebsmodells}

Die Steuerung eines digitalisierten Vertriebskanals am Beispiel eines Informations- und Kommunikationstechnologieunternehmen

Dipl.-Kfm. Christian Huck, Dr. Sven Gerdes und Martin Krömer, M.Sc.

\section{End-to-End-Prozessorientierung als Treiber der digitalen Transformation}

Vorteile junger Unternehmen und digitaler Geschäftsmodelle und welche Chancen daraus auch für etablierte

Dipl.-Kfm. Jan Georg Sodies und Tobias Meyer, M.A.

Steuerungsgrößen für digitale Geschäftsmodelle in Produktionsunternehmen

Benötigen wir neue Steuerungsgrößen?

Garlef Hupfer, M.Sc., Benedict Wytopil, M.Sc. und Linda Gebhardt, M.Sc.

Gezielte Verwendung von Farben im Management Reporting

Wie Sie im Reporting Farbe in die graue Welt der Daten bringen

Garlef Hupfer, M.Sc. und Felicitas Heering, M.Sc.

\section{AKTUELL}

Drohende Zahlungsunfähigkeit im Insolvenzreife-Monitoring

Prof. Dr. Alexander Baumeister, Fabio Britz, M.Sc. und Tobias Kochems, M.Sc.

\section{COMPACT}

Instandhaltungssteuerung in der Industrie 4.0

Vom Kostentreiber zum Werttreiber

Martin Eisenmann, M.A., Johanna Holzhaver-Knocks, M.Sc., und Daniel Silveira Pereira, M.Sc.

\section{WISSEN}

Implementierung von Predictive Analytics im Forecasting

Algorithm Aversion und weitere Herausforderungen

Prof. Dr. Gero Holthoff und René Decher, M.A. 
Stand des IT-Einsatzes im Controlling

Prof. Dr. Thorsten Knauer, Dr. Nicole Nikiforow und Dr. Sebastian Wagener

\section{IM DIALOG}

Gründer-Programm in einem Familien-Unternehmen

Dr. Goran Sejdic, Dr. Marcel Gebhardt und Prof. Dr. Dr. h.c. mult. Péter Horváth

\section{CONTROLLING UND DIGITALISIERUNG}

Stand der Digitalisierung des Controllings in Familienunternehmen

Prof. Dr. Martin R. W. Hiebl und David I. Pielsticker, M.Sc.

\section{LEXIKON}

Customer-Value

Wertversprechen in digitalen Geschäftsmodellen

Sebastian Kasselmann, M.Sc.

\section{LITERATUR-TIPPS}

Grundlagenliteratur zu digitalen Geschäftsmodellen/Fachbuch-Test

\section{VERANSTALTUNGEN \\ Nachlese/Vorschau/Kongresse · Seminare · Workshops}

Vorschau auf Heft 1/2021 und Impressum

Das aktuelle Heft · Archiv · Newsletter: www.zeitschrift-controlling.de

Aus Gründen der leichteren Lesbarkeit wird in der vorliegenden Zeitschrift die gewohnte männliche Sprachform bei personenbezogenen Substantiven und Pronomen verwendet. Dies impliziert jedoch keine Benachteiligung anderer Geschlechter, sondern soll im Sinne der sprachlichen Vereinfachung als geschlechtsneutral zu verstehen sein.

\section{> DIE HERAUSGEBER}

Die Controlling gehört zu den wichtigsten Zeitschriften für Fach- und Führungskräfte im Finanz- und Rechnungswesen von Unternehmen und öffentlichen Institutionen. Sie liefert fundierte und anwendungsorientierte Beiträge für alle Controlling-Bereiche, zu allen Branchen und für unterschiedliche Unternehmensgrößen. Sie wird herausgegeben von:

Prof. Dr. Dr. h.c. mult. Péter Horváth, IPRI International Performance Research Institute/Universität Stuttgart Prof. Dr. Thomas Reichmann, Technische Universität Dortmund und $\mathrm{ClC} \mathrm{GmbH,} \mathrm{Dortmund}$

Prof. Dr. Ulrike Baumöl, Universität Liechtenstein

Prof. Dr. Andreas Hoffjan, Lehrstuhl Unternehmensrechnung und Controlling, Technische Universität Dortmund

Prof. Dr. Klaus Möller, Lehrstuhl Controlling/Performance Management, Universität St. Gallen

Prof. Dr. Burkhard Pedell, Lehrstuhl für Controlling, Universität Stuttgart 